\title{
A Case of External Auditory Canal Sebaceous Carcinoma: Literature Review and Treatment Discussion
}

\author{
Robert Saadi $^{a}$ Michael Pennock ${ }^{a}$ b Aaron Baker ${ }^{a}$ Huseyin Isildak ${ }^{a}$ \\ aDepartment of Otolaryngology, Head and Neck Surgery, College of Medicine, \\ The Pennsylvania State University, Hershey, PA, USA; ${ }^{b}$ Department of Surgery, \\ Albert Einstein College of Medicine, Montefiore Medical Center, Bronx, NY, USA
}

\section{Established Facts}

- Sebaceous carcinoma is a rare type of skin cancer overall and there are less than 15 known cases in the literature originating from the external auditory canal (EAC). As such, the present case is a discussion of a rare tumor in an exceedingly rare location.

- Surgical excision is the preferred treatment for sebaceous carcinoma. Recurrence is high and radiation may be indicated based on tumor extension or metastasis.

\section{Novel Insights}

- Recommendations for the extent of surgical excision of primary EAC malignancies are in place specifically for squamous cell carcinoma, but can be reasonably applied to sebaceous carcinoma, as in the present case. Limited resection without recurrence was possible using these guidelines.

- The patient's pathology demonstrated favorable prognostic features. As such, close observation was appropriate and the patient had no recurrence for over 3 years.

\section{What Is It about?}

Sebaceous neoplasms are a rare type of skin cancer that may occur in any area with sebaceous glands. The outer third of the external auditory canal is a potential site, given the presence of these glands. However, documentation of the tumor originating in this location is limited to scarce case reports. In this location, malignancies can invade the temporal bone, typically requiring en bloc resection and radiation therapy. We describe a case managed with limited surgical excision based on criteria for primary squamous cell carcinoma of the external auditory canal with only close observation following surgery. 


\title{
Keywords
}

Otology $\cdot$ Carcinoma $\cdot$ Auditory canal $\cdot$ Ablation

\begin{abstract}
Sebaceous neoplasms are a rare type of skin cancer that may occur in any area with sebaceous glands, including the outer third of the external auditory canal. However, documentation of the tumor originating in this location is limited to scarce case reports. In this location, malignancies can invade the temporal bone, typically requiring en bloc resection and radiation therapy. We describe a case managed with limited surgical excision based on criteria for primary squamous cell carcinoma of the external auditory canal with only close observation following surgery. Margins were free of disease following excision and histology demonstrated a favorable prognosis. The patient was disease free after a follow-up period of over 3 years postoperatively. A review of relevant literature is utilized to discuss clinical characteristics, staging, treatment, and prognosis to assist in clinical decision making for these patients.

(C) 2020 The Author(s)

Published by S. Karger AG, Basel
\end{abstract}

\section{Introduction}

Sebaceous neoplasms represent $0.7 \%$ of skin cancers, occurring primarily in areas of higher density of sebaceous glands, including the eyelids, scalp, neck, and face [1]. Sebaceous carcinoma has a predilection for head and neck locations, with $38.7 \%$ periorbital, $40.8 \%$ extraorbital head and neck, and $19.9 \%$ other (e.g., trunk, limbs, etc.) [1, 2]. The overall incidence of sebaceous carcinoma is 1 in 1,000,000 persons per year [1,2].

The external auditory canal (EAC) consists of two sections, separated at the isthmus. The anteroinferior and posteroinferior tympanic bone, superior squamous temporal bone, and posterior mastoid bone form the inner bony canal, covered with a thin layer of stratified squamous epithelium. The cartilaginous outer third of the EAC is lined with stratified squamous epithelium as well as dermal adnexa, which may give rise to glandular neoplasms [2]. There are less than 15 known cases in the literature originating from the EAC. Given the rarity of the disease and the potential implications on surgical extent based on location, our goal was to discuss clinical characteristics, staging, treatment, and prognosis in general and in relation to the present case to assist in clinical decision making for these patients.

\section{Case Presentation}

A 79-year-old woman with history of immunocompromise and left neck squamous cell carcinoma (SCC) presented with an EAC pedunculated mass, otorrhea, and unilateral conductive hearing loss over 5 months. She denied otalgia, facial nerve paralysis, fever, weight loss, or night sweats. Outside biopsy revealed a well-differentiated atypical pilosebaceous neoplasm. Imaging revealed increased soft tissue in the EAC on computed tomography (CT) imaging without bony extension (Fig. 1). The patient underwent EAC wide local excision, canaloplasty, and temporalis fascia graft reconstruction. There was no evident cartilaginous involvement. The final pathology revealed a well-differentiated sebaceous carcinoma without perineural or vascular invasion and clear circumferential margins $>5 \mathrm{~mm}$. Observation and close follow-up were recommended. Imaging performed after 3 months was negative for recurrent or persistent cancer (Fig. 2). Clinically the patient has remained disease free for more than 3 years postoperatively. 
Fig. 1. Preoperative CT showing an abnormal soft tissue mass in the left cartilaginous canal without obvious extension into adjacent soft tissue or erosion of the temporal bone.

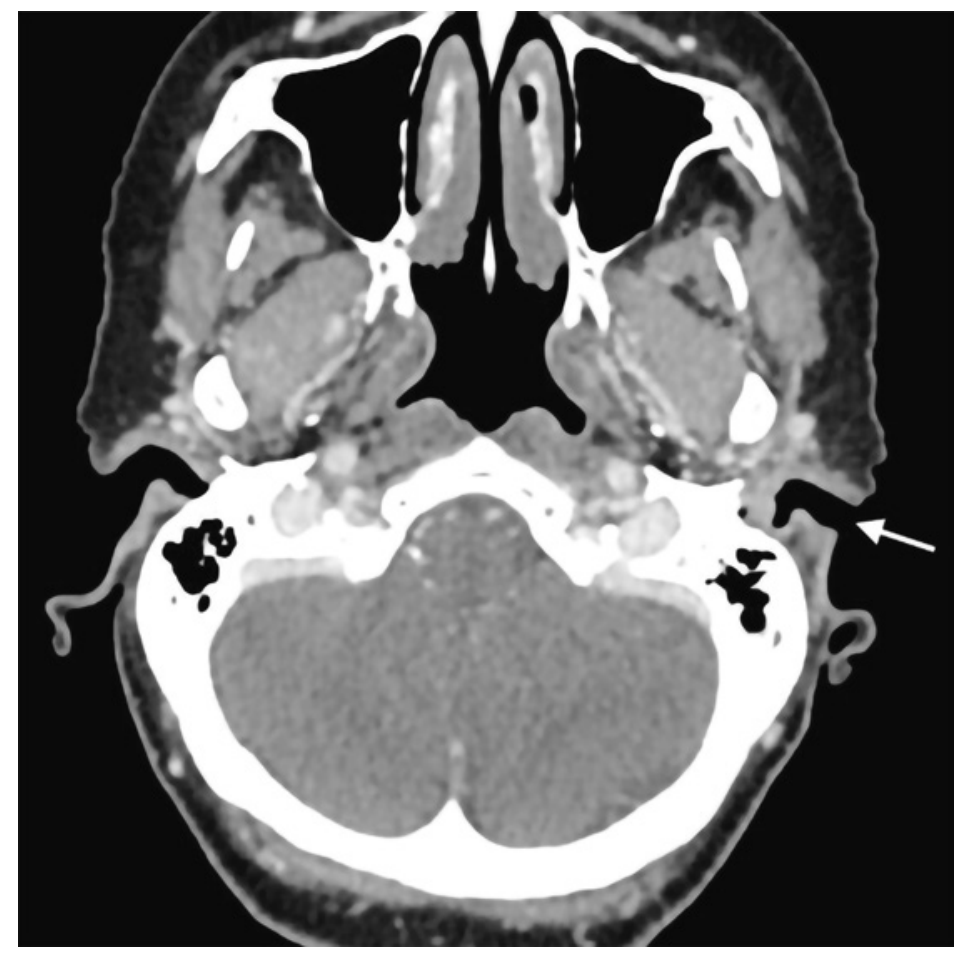

\section{Discussion}

\section{Clinical Presentation}

Although EAC sebaceous carcinoma may be associated with hearing loss, otorrhea, or blockage, the tumor is generally asymptomatic and many patients may present late with locally advanced disease [3]. Tumors appear papular, yellow, red, or skin colored [3]. Differential diagnosis of an EAC mass should include cholesteatoma, polyps, sebaceous nevi, ceruminous malignancy, SCC, keratosis obturans, basal cell carcinoma (BCC), Merkel cell carcinoma, melanocytic lesions, cavernous hemangioma, otitis externa, and metastases [2]. Benign sebaceous nevi have been noted to progress to sebaceous cell carcinoma [4].

\section{Muir-Torre Syndrome Screening}

Associations with sebaceous carcinoma include Muir-Torre syndrome (MTS), irradiation, immunosuppression, and familial retinoblastoma [5, 6]. Thirty percent of patients with MTS develop carcinoma [6]. MTS is an autosomal dominant variant of Lynch syndrome manifesting as sebaceous neoplasm and visceral malignancy [5]. The affected genes are MLH1, MSH2, and MSH6 in mismatch repair, resulting in microsatellite instability and tumor predisposition. Epidermal growth factor receptor, vascular endothelial growth factor, and tumor suppressor (p53) mutations have been found in cases with intact mismatch repair [7]. Sebaceous neoplasms should prompt MTS work-up: genotyping, geneticist referral, and monitoring for visceral malignancy [8]. Given our patient's advanced age, lack of personal history of visceral malignancy, lack of family history of Lynch syndrome or MTS, and single sebaceous neoplasm, MTS was very unlikely and further MTS work-up was deferred.

\section{Pathology and Histology}

Sebaceous tumors are one of three overlapping types: sebaceous adenoma, BCC with sebaceous differentiation, and sebaceous carcinoma. Specific sebaceous carcinoma antibodies 
Fig. 2. Three-month postoperative MRI showing the site of excision just medial to tragal cartilage without evidence of disease.

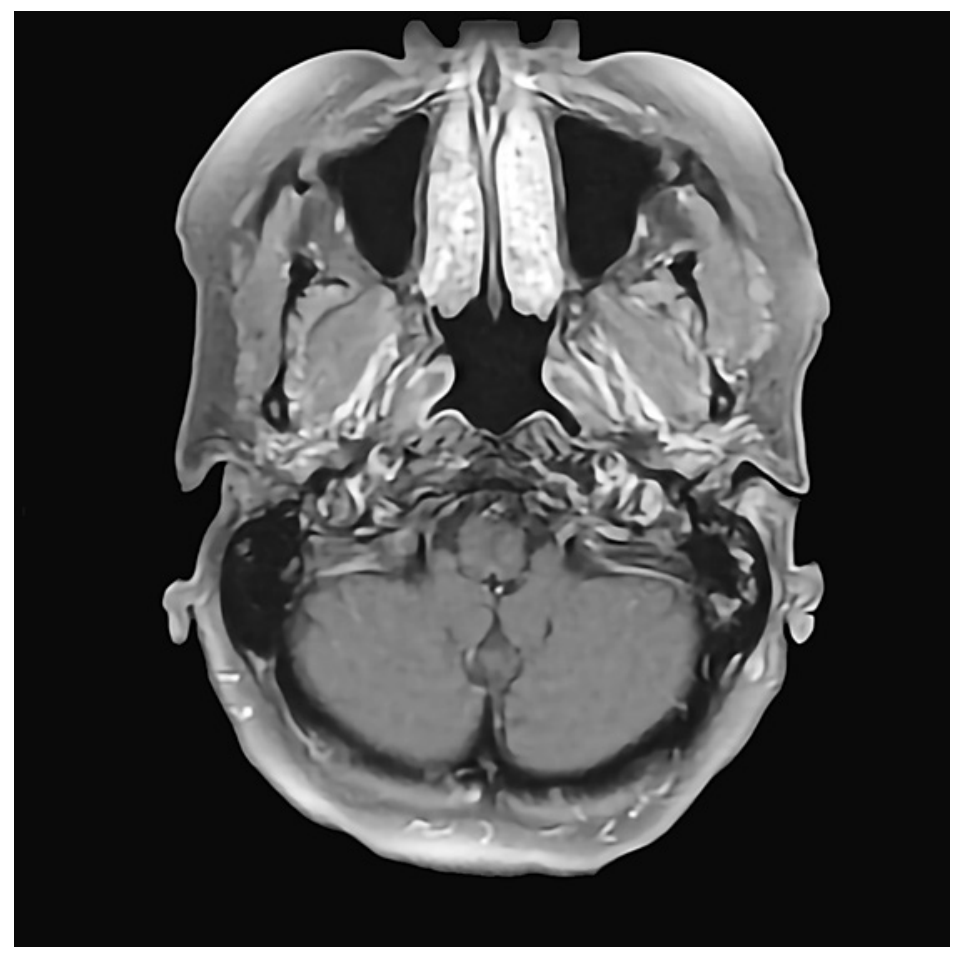

include: AE1/AE3, EMA, adipophilin, perilipin, p53, Ki-67, BCA-225, Cam 5.2, and Ber EP4, which can distinguish BCC from sebaceous carcinoma with $100 \%$ specificity and sensitivity $[9,10]$. Recently, alpha/beta hydrolase domain-containing protein 5 (ABDH-5) and progesterone receptor membrane component-1 (PGRMC-1) have been used to differentiate the benign sebaceous adenomas from sebaceous carcinoma [9]. Sebaceous adenomas appear as dermal cribriform basophilic cells and sebocyte clusters, in contrast to nuclear changes and infiltration seen in sebaceous carcinoma [9]. There are four sebaceous carcinoma histologic patterns: lobular, comedo, papillary, and mixed, and cells can be well, moderately, or poorly differentiated, with markers of poor prognosis being pagetoid spread, multicentricity, tumor size $>10 \mathrm{~mm}$, and invasion of vascular, lymphatic, and perineural structures $[1,9,10]$. There is great interobserver variability among pathologists' diagnoses of sebaceous neoplasms $[1,8,10]$. The patient's pathology demonstrated a well-differentiated sebaceous carcinoma with epithelioid proliferation, sebaceous markers, nuclear pleomorphism, and hyperchromatism, lacking markers of poor prognosis. The tumor's well-differentiated nature meant wide local excision was sufficient.

\section{Staging}

Sebaceous carcinomas are staged in location-dependent fashion via AJCC TNM guidelines for non-melanoma skin cancers $[10,11]$. Extraorbital sebaceous carcinoma staging would typically be classified as a cutaneous carcinoma of the head and neck [11]. However, extraorbital sebaceous carcinoma TNM staging has not been validated for predicting cancer-specific mortality $[10,11]$. Studies have reported regional metastasis rates of $2.4-12 \%[10,11]$. The Pittsburgh staging system, originally developed for SCC, has been adopted for EAC carcinoma to attempt to establish staging guidelines, as there is no consensus among staging systems $[10,12]$. Recent studies have shown that the University of Pittsburgh staging system carries higher prognostic accuracy than the latest AJCC staging system, with Pittsburgh T classification being an independent predictor of the overall survival rate, thus verifying that the system carries prognostic importance [12]. The current patient was staged T1N0M0 based 
on the Pittsburgh system, thus wide local excision was sufficient, as it was a T1 tumor limited to the EAC showing clear margins by histopathology upon removal, without bony erosion or soft-tissue extension $[10,13]$. Anterior wall erosion of the auditory canal (T2) would indicate the need for superficial parotidectomy and en bloc lateral temporal bone resection or subtotal temporal bone resection $[10,12,13]$. No radiation or neck dissection was indicated according to the Pittsburgh system, as there were no intraoperative findings suggestive of extensive disease, and no nodal spread was detected in the neck on imaging or exam $[10,12,13]$.

\section{Imaging}

CT imaging is the first-choice diagnostic modality [13]. Magnetic resonance imaging (MRI) is useful in delineating soft-tissue margins, infiltration, and vascular encasement [13]. Few studies have addressed imaging standards for EAC sebaceous carcinoma. Palpable lymphadenopathy should prompt consideration for lymph node dissection [14]. In the EAC, the fissures of Santorini and lymphatics may facilitate easy local and regional spread. Ninety percent of primary temporal bone malignancies have an EAC origin. As such, EAC masses should prompt temporal bone assessment with CT imaging $[1,11]$. Advanced imaging should be obtained for aggressive features such as perineural invasion, poorly differentiated histology, or size $>20$ mm (extraorbital sebaceous carcinoma AJCC T2) $[13,14]$. Due to its aggressive nature, sebaceous carcinoma should prompt vigilant follow-up, with AJCC T3 or higher requiring chest $\mathrm{X}$-ray, regional node ultrasound, and bone scan every 3 months after diagnosis for 1 year, then every 6 months for another year, and then once a year $[13,14]$. Our patient did not have highrisk cancer, thus preoperative CT scan was sufficient, with follow-up MRI.

\section{Treatment}

Surgical excision is preferred for diagnosis and treatment despite high postoperative recurrence via pagetoid spread (19-37\%), while radiation may be indicated based on tumor extension or metastasis [11]. Local recurrence rates have declined in the last decade (4-28\%) from increased awareness and earlier diagnosis $[3,9]$. If the tumor invades the temporal bone, the patient requires en bloc EAC and temporal bone resection, followed by radiotherapy for recurrence [11]. Tumor status, clinical status, hearing preservation, and facial nerve involvement influence the extent of skull base approaches. Radiation alone carries higher mortality compared to surgical excision, and thus is an adjunct for high-risk features, recurrent tumor, or positive surgical margin [11]. A combination of radical surgery and radiation therapy has provided better outcomes than surgery alone $[9,11]$. Microvascular free flap and temporalis muscle flap were most often used for reconstruction [15]. The disease-free 5-year survival of temporal bone cancers was $42-44 \%[1,9,11,15]$. Reports detailing the use of chemotherapy are limited. Platinum and 5-fluorouracil most often trigger a treatment response [15]. The current patient's lesion involved EAC soft tissue without invasion, thus complete tumor excision was sufficient without radical surgery or radiation.

\section{Prognosis}

Historically, periorbital sebaceous carcinomas carry a poor prognosis, with a metastasis rate of $14-28 \%$, and a mortality of $18-30 \%[9,10]$. Recent studies show lower mortality rates $(3-6.7 \%)[2,9]$. Sebaceous carcinomas limited to the EAC are less likely to invade regional lymph nodes than primary preauricular carcinomas that secondarily invade the ear canal $[2$, $10,13]$. Significant predictors of reduced survival are metastasis at diagnosis, a non-EAC primary site, and pretreatment facial paralysis [11]. Regular follow-up is required. The overall 5-year survival rate and the 5-year disease-free survival for malignancy involving the temporal bone are 58.0 and $54.9 \%$, respectively, with EAC-limited disease carrying better survival than periauricular primary tumors or temporal bone invasion [15]. 


\section{Conclusion}

EAC sebaceous carcinoma is a rare, aggressive neoplasm with unclear pathophysiology. Diagnosis requires pathology, IHC, family history, MTS work-up, imaging, lymphadenopathy assessment, and SLN biopsy in select cases. The incidence of metastasis is declining. Surgical excision is the primary treatment, with adjunct radiotherapy preferred over chemotherapy. Extraorbital sebaceous carcinoma TNM staging has not been validated. Given the high recurrence rate when the cancer occurs in other sites on the body, an evidence-based approach to sebaceous carcinoma of the EAC is challenging and treatment relies mainly on data for the more commonly encountered SCC of the EAC. The present case demonstrates that limited surgical excision based on the early stage by the Pittsburgh T classification and close observation given the favorable histologic prognosis is appropriate for this exceedingly rare tumor when located in the EAC.

\section{Statement of Ethics}

The present article complies with guidelines for human studies. The study was approved by the Institutional Review Board at Penn State Medical Center. Written informed consent was obtained from the subject preoperatively for publication.

\section{Disclosure Statement}

The authors have no conflicts of interest to declare.

\section{Funding Sources}

No funding was acquired for this work.

\section{Author Contributions}

R.S. and H.I. - manuscript conception, collection of data, synthesis of data, manuscript construction, manuscript revisions, approval for submission. M.P. and A.B. - manuscript conception, collection of data, synthesis of data, manuscript construction, approval for submission.

\section{References}

1 Bailet JW, Zimmerman MC, Arnstein DP, Wollman JS, Mickel RA. Sebaceous carcinoma of the head and neck. Case report and literature review. Arch Otolaryngol Head Neck Surg. 1992 Nov;118(11):1245-9.

2 Gidley PW, Thompson CR, Roberts DB, DeMonte F, Hanna EY. The oncology of otology. Laryngoscope. 2012 Feb;122(2):393-400.

3 Shields JA, Demirci H, Marr BP, Eagle RC Jr, Shields CL. Sebaceous carcinoma of the ocular region: a review. Surv Ophthalmol. 2005 Mar-Apr;50(2):103-22.

4 Crabtree JA, Britton BH, Pierce MK. Carcinoma of the external auditory canal. Laryngoscope. 1976 Mar;86(3): 405-15.

5 Cohen PR, Kohn SR, Kurzrock R. Association of sebaceous gland tumors and internal malignancy: the MuirTorre syndrome. Am J Med. 1991 May;90(5):606-13.

6 Cohen PR, Kohn SR, Davis DA, Kurzrock R. Muir-Torre syndrome. Dermatol Clin. 1995 Jan;13(1):79-89. 
7 Shalin SC, Sakharpe A, Lyle S, Lev D, Calonje E, Lazar AJ. p53 staining correlates with tumor type and location in sebaceous neoplasms. Am J Dermatopathol. 2012 Apr;34(2):129-35.

8 Orta L, Klimstra DS, Qin J, Mecca P, Tang LH, Busam KJ, et al. Towards identification of hereditary DNA mismatch repair deficiency: sebaceous neoplasm warrants routine immunohistochemical screening regardless of patient's age or other clinical characteristics. Am J Surg Pathol. 2009 Jun;33(6):934-44.

9 Tryggvason G, Bayon R, Pagedar NA. Epidemiology of sebaceous carcinoma of the head and neck: implications for lymph node management. Head Neck. 2012 Dec;34(12):1765-8.

10 Moody SA, Hirsch BE, Myers EN. Squamous cell carcinoma of the external auditory canal: an evaluation of a staging system. Am J Otol. 2000 Jul;21(4):582-8.

11 Kim CS, Suh MW. Skull base surgery for removal of temporal bone tumors. Acta Otolaryngol Suppl. 2007 Oct; 127(558):4-14.

12 Morita S, Mizumachi T, Nakamaru Y, Sakashita T, Kano S, Hoshino K, et al. Comparison of the University of Pittsburgh staging system and the eighth edition of the American Joint Committee on Cancer TNM classification for the prognostic evaluation of external auditory canal cancer. Int J Clin Oncol. 2018;23:1029-37.

13 Horowitz SW, Leonetti JP, Azar-Kia B, Fine M, Izquierdo R. CT and MR of temporal bone malignancies primary and secondary to parotid carcinoma. AJNR Am J Neuroradiol. 1994 Apr;15(4):755-62.

14 Kyllo RL, Brady KL, Hurst EA. Sebaceous carcinoma: review of the literature. Dermatol Surg. 2015 Jan;41(1): $1-15$.

15 Manolidis S, Pappas D Jr, Von Doersten P, Jackson CG, Glasscock ME 3rd. Temporal bone and lateral skull base malignancy: experience and results with 81 patients. Am J Otol. 1998 Nov;19(6 Suppl):S1-15. 